\title{
Model for the Reaction-Rate-Limited Dissolution of Solids with Etch-Rate Heterogeneities
}

\author{
Eric'A. Robertson III and H. Scott Fogler \\ Dept. of Chemical Engineering, University of Michigan, Ann Arbor, MI 48109
}

\begin{abstract}
The coalescence of isotropic etch pits observed in the dissolution of semiconductor substrates is studied using a discrete model for the evolution of the surface under reaction-rate-limited conditions. The model discretizes the solid into cubic elements and repetitively applies dissolution rules to the individual elements. The rate of mass removal is based on the number and arrangement of the element's exposed faces and the specified reaction-rate parameters. Detailed knowledge of the surface normal is not required. The model shows that even at moderate etch pit densities, the effects of the coalescence do not significantly alter the trends observed for noncoalescing etch pits.
\end{abstract}

\section{Introduction}

The evolution of surfaces is a topic that arises in a large number of fields. Examples include deposition, corrosion, erosion, combustion of solids, the growth of bacterial cell colonies, and evaporation (see Cohen, 1980; Qian et al., 1990; Meakin, 1987; Meakin et al., 1993; Eden, 1958; Weeks and Gilmer, 1978). Microelectronics in particular has seen a steady interest in the evolution of surfaces under growth conditions due to the importance of deposition in the fabrication of solid-state devices (Cooke and Harris, 1989; Ikegawa and Kobayashi, 1989; Cale and Raupp, 1990; Watanabe and Komiyama, 1990; Wulu et al., 1991; Dew et al., 1992; Singh and Shaqfeh, 1993). The study of the growth or dissolution of many solids is complicated by either a complex geometry or a rate that varies with position and/or the orientation of the surface. Causes of such difficulties include, but are not limited to, (1) complicated geometries; (2) etch rates that are functions of position, orientation, or a time-varying property; and (3) situations that couple the etch rate at the surface to properties of the bulk of the solid. Examples include the crystallographic etching of semiconductors, the dissolution of random binary mixtures, and the combustion of solid mixtures of fuel and oxidizers. In most cases, conditions such as these are too complex to allow a direct, analytical description of the dissolution process. Therefore alternative methods must be devised.

One approach is to view the surface as a continuous boundary that moves as material is deposited or removed. The boundary is presumed to move in a direction parallel to the surface normal. However, problems arise when the sur-

Correspondence concerning this article should be addressed to H. S. Fogler. face tangent cannot be rigorously defined, such as at corners and edges. Also the mass flux can depend on the spatial coordinates, the surface orientation, local and bulk properties, and time. Analytical descriptions are available for only the simplest cases, and numerical solutions become cumbersome and time-consuming (Hubbard and Antonsson, 1994; Séquin, 1992; Jaccodine, 1962; Thurgate, 1991; Katardjiev, 1989).

Another approach, used more frequently for vapor-phase deposition than etching, is to follow each particle of the mass flux and apply kinematic laws to determine where it impacts the surface and how likely it is to stick. Clearly such an approach is best suited for gas-phase reactions at low pressure where the reactant molecules undergo very few collisions before reaching the surface of the semiconductor. Monte Carlo methods are often used to simulate the flight of the particles responsible for the surface evolution (Cooke and Harris, 1989; Ikegawa and Kobayashi, 1989; Yuuki et al., 1989; Cale and Raupp, 1990; Wulu et al., 1991). Such an atomistic approach can handle complex surfaces and a large variety of physical processes, but becomes intractable when the system has millimeter-scale dimensions or the etch is performed in a liquid phase.

Recently, Robertson and Fogler observed that etch pits could be induced in semiconductor layers using a combination of applied electric field and surface illumination (Robertson and Fogler, 1996; Robertson, 1995). In this instance, the pits are the result of local variations in the etch rate of a semiconductor epilayer. The variations in the etch rate lead to the premature exposure of the substrate to the etchant and the subsequent formation of an etch pit in the substrate. The isotropic growth of an individual pit was modeled quan- 
titatively using an analytical expression for the surface area as a function of time, with a delay incorporated to account for the time needed to etch through the epilayer (Robertson and Fogler, 1996). It was shown that the length of the delay for a particular pit was distributed over a range ur values, which leads to distribution in the sizes of the etch pits even though the initial defect area is the same for all defects. Using this model to describe a system of several pits is limited to cases where the pits grow isotropically and independently of one another.

Eventually, the pits coalesce and the mass flux decreases because the surface area of the coalesced pits is less than that of the same pits growing independently. What remains to be determined is how do systems of etch pits behave as they grow and coalesce. In these systems, the pits occur at random over the surface. A description of the behavior of randomly arranged pits over a long period of time was not possible due to the interaction of the pits. A scanning electron micrograph of the coalescence of two pits is shown in Figure 1A. Figure 1B depicts the isotropic growth of a single etch pit under a slowly etching semiconductor layer (Robertson and Fogler, 1996).

This article presents a method of describing the evolution of a macroscopic, solid surface without detailed information about the local surface tangents. The method can be applied to a wide range of geometries and can accommodate variations in etch rate due to the orientation, position, time, and constraints that arise due to communication from one region to another. The model discretizes the solid object, or portion of a solid surface, into small, homogeneous, cubic elements. Dissolution rules are applied to each element on the surface. A portion of the element's volume is removed commensurate with the characteristics of the dissolution reaction and the length of the time step. This article first describes the details of the development of the model, and then presents the model predictions compared to the analytical results for the isotropic dissolution of a single etch pit. Finally, the model is used to study the behavior of heterostructures with a distribution of pits resulting from random etch-rate heterogeneities similar to those observed experimentally and reported previously (Robertson and Fogler, 1996).

\section{Model Development}

There are three aspects to the development of this model: (1) the discretization of the domain and its geometry into individual elements; (2) formulation of the equations for the dissolution of an individual element and the method of calculating the surface profile; and (3) the establishment of the rules governing the mass flux for individual elements over unit time steps.

The solid-on-solid (SOS) model described by Weeks and Gilmer (1978) also discretizes the solid into elemental cubes, but on an atomic scale. The SOS model is limited because it only allows growth or dissolution from the top face. Therefore, no consideration is given to the consequences of growth or dissolution from other exposed faces. The result is that each element must reside on top of another element, precluding any type of undercutting or lateral dissolution.

\section{Geometry}

In this application the domain is a rectangular prism with

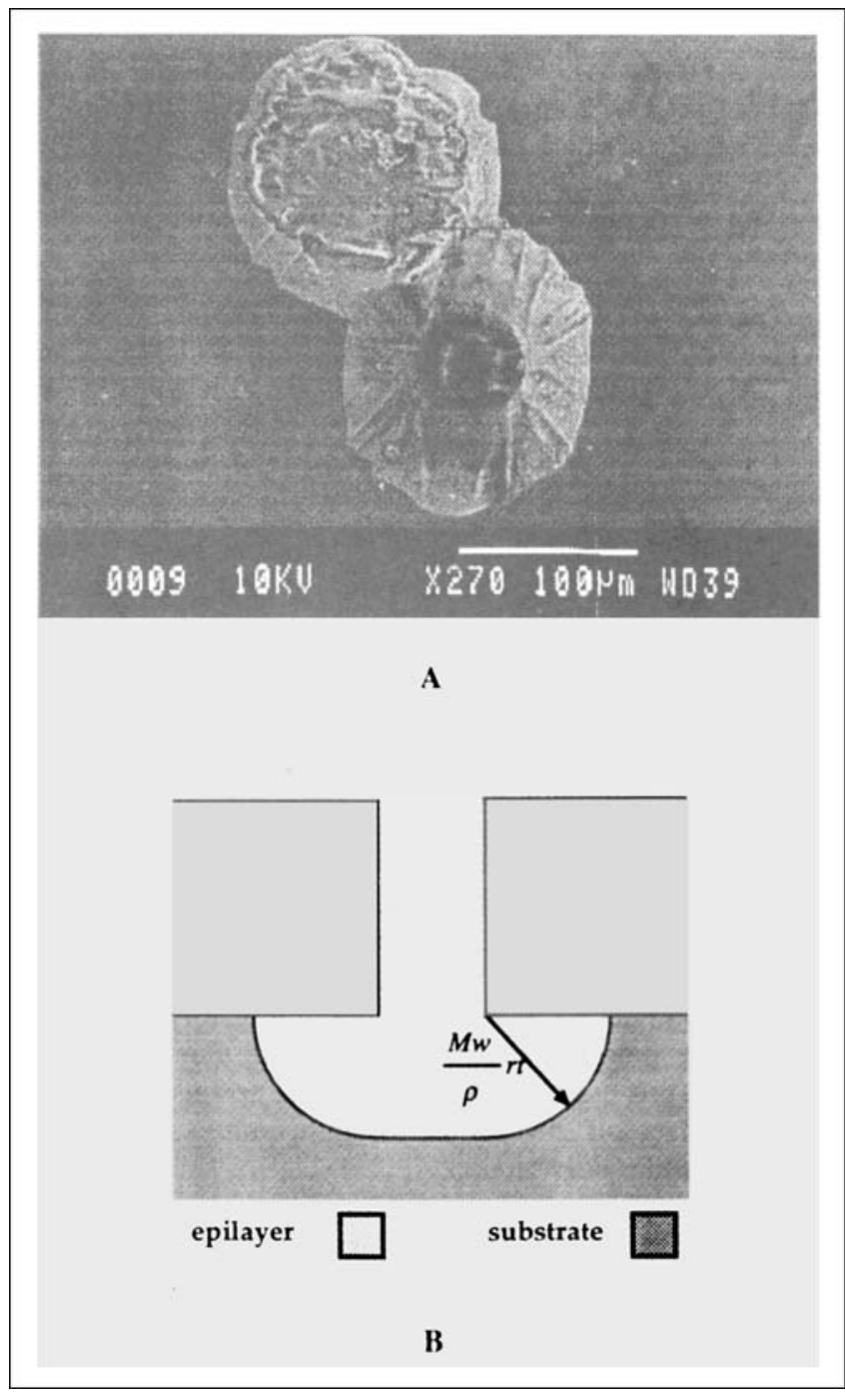

Figure 1. (A) Scanning electron micrograph of two coalesced pits in a GaAs substrate; (B) Representation of a pit.

The time denoted by $t$ is the time since the initial exposure of the substrate.

length $L$, width $W$, and depth $D$, not necessarily equal. However, the approach can be implemented for any arbitrary solid geometry. The six faces of the prism are denoted by up, down, north, south, east, and west. The up face is where the dissolu. tion begins; the down face is nonetching by definition. Periodic boundary conditions are applied to the north-south and the east-west faces, respectively.

The domain is then subdivided into cubic elements of equal volume with a characteristic edge length $s$. Each element has homogeneous material properties: density, composition, molecular weight, and so on. The domain is composed of $n_{k}$ levels, each with $n_{i}$ rows and $n_{j}$ columns of elements. The six faces of each element are denoted up, down, north, south, east, and west. The up face for an element is the face whose outward normal vector points to the up face of the domain prism. The remaining faces of the volume element are similarly defined. 


\section{Dissolution of a single element}

The evolution of the surface profile is predicted based on the initial geometry of the solid and on dissolution rules applied to the individual cubic elements making up the solid. For a cubic element, the initial change in mass over a small time increment is approximately given by

$$
\Delta m=-N s^{2} M w r \Delta t,
$$

where $m$ is the mass of the element; $\Delta t$ is the time increment; $M w$ is the molecular weight; $s$ is the edge length of the cubic element; $N$ is the number of exposed faces; and $r$ is the molar flux, which, for the purposes of this work, is assumed constant for each face of an individual element. A positive $r$ represents mass leaving the volume element.

As the element dissolves, the exposed area decreases and is no longer equal to $N s^{2}$. However, it may be represented by a quantity $K s^{2}$, where $K$ is an effective number of exposed faces:

$$
\Delta m=-K s^{2} M w r \Delta t .
$$

$K$ 's depending only on the number and arrangement of an element's exposed faces provide very good representations of the dissolution trends reported previously by Robertson and Fogler (1996). However, other factors may also be considered in assigning values to $K$, for example, the history of the element or the present mass of the element. For clarity, the subscript $N, a$ is used to refer to a specific arrangement, $a$, of $N$ exposed faces. The rules for assigning values to $K_{N, a}$ are addressed in the next section.

The average mass flux and the total mass transferred per unit area are computed from the mass transferred from each exposed element. First, the total mass transferred during a single time step, $\Delta M$, is given by the sum of masses transferred from each exposed element during that time step:

$$
\Delta M=\Sigma \Delta m(t),
$$

where the summation is over all of the exposed elements. The average mass flux over that time step is

$$
R=-\frac{\Delta M}{\Delta t A_{0}},
$$

where the initial surface area is used as the normalizing area. The total mass transferred per unit initial area, $M$, is

$$
M=\frac{1}{A_{0}} \Sigma \Delta M,
$$

where the summation in this case is carried over the sequence of time steps.

\section{Dissolution rules}

Intuitively, it is expected that the number of faces exposed to the etchant is important in determining $\Delta m$ for an element. However, in addition to the number of exposed faces, the arrangement of those faces must also be considered be-

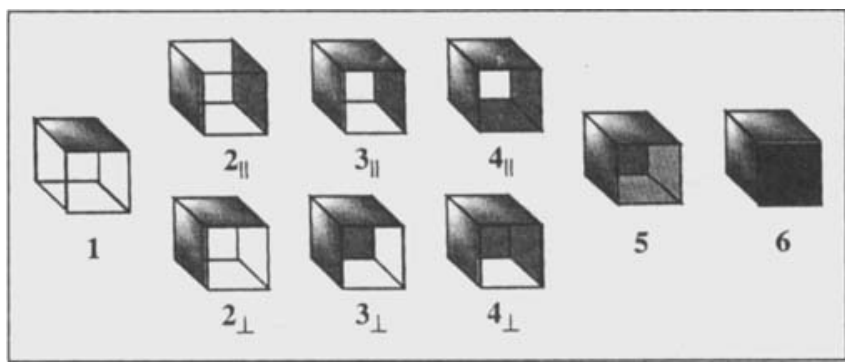

Figure 2. Arrangements of one to six exposed (shaded) faces.

fore the dissolution rules can be established. In general, there are multiple ways to arrange a given number of exposed faces. There are six ways to arrange one exposed face and five unexposed faces on a cube, but those arrangements are degenerate in this application since all faces have the same characteristic rate. There are two nondegenerate ways to arrange two, three, and four exposed faces. For example, two exposed faces can be either parallel to each other or perpendicular to each other. Five and six exposed faces can be arranged in only a single nondegenerate fashion. Figure 2 shows the nondegenerate arrangements for one to six exposed faces.

The arrangements of the exposed faces can be separated into two different configurations: those that have one or more pairs of parallel faces exposed to the etchant and those that do not. Elements in the former configuration are referred to as kinks $\left(2_{\|}, 3_{\|,}, 4_{\perp, \|}, 5\right.$, and 6$)$, while those in the latter configuration are referred to as planar $\left(1,2_{\perp}\right.$, and $\left.3_{\perp}\right)$. With the exception of the $2_{\|}$arrangement, kinks tend to be very rare. Therefore the overall results are not strongly dependent on the values assigned to $K_{N, a}$ for these elements.

The values assigned to $K_{N, a}$ are developed based on a set of rules. The rules used in this work are:

1. The effective exposed area for a kink element is based on the time-averaged area of the element over its lifetime.

2. The effective exposed area for a planar element is determined by projecting the exposed faces onto a single plane. The plane used is the one that provides the greatest projected area.

For example, $2 \|$ has two parallel exposed faces and is therefore a kink element; it falls under Rule 1 . The exposed area is $2 s^{2}$ for the lifetime of the element, and $K_{2, \|}$ is therefore 2 (see Figure 3). On the other hand, $2_{\perp}$ has two perpendicular exposed faces and falls under Rule 2 . Clearly the maximum projected area in this case will be greater than $1 s^{2}$ but less than $2 s^{2}$. It can be easily shown that the plane upon which to project the areas of the exposed faces that gives the maximum area is the one that lies at a $45^{\circ}$ angle to both faces. In that case, the projected area is $\sqrt{2} s^{2}$. Thus $K_{2, \perp}$ is $\sqrt{2}$. Table 1 lists the values of $K_{N, a}$ for all nine arrangements, and a detailed derivation of these values is presented in the Appendix. Using these rules, the profile of the dissolving surface and the mass removal rate can be predicted as a function of time for complicated systems without detailed knowledge of the surface orientation.

The dissolution rules are based on the number and arrangement of the exposed faces. Other rules could be proposed to incorporate more information into the model. For example, one might also include the present volume or the 


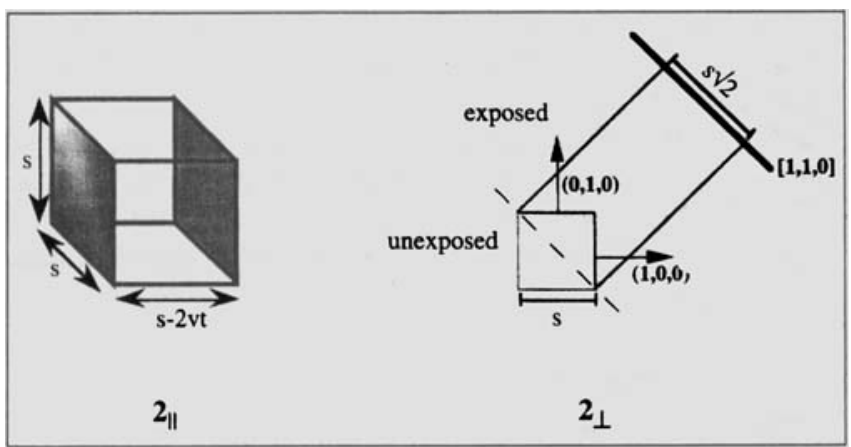

Figure 3. Geometric interpretation of values for $K_{2, \mid z}$ and $K_{2, \perp}$.

Here, $s$ is the initial edge length, $\nu$ is the velocity of the etch, and $t$ is the time the face has been exposed.

history of the element in determining the amount of material to remove from the element. Additionally, the orientation of the exposed elements was not considered in these rules because the pits observed did not indicate a strong dependence of etch rate on orientation. Considering only the arrangement of exposed faces quantitatively describes systems with these etch-rate heterogeneities.

\section{Dissolution of Isotropic Etch Pits}

This section presents the model results compared to the dissolution of a test structure. The test structure chosen is a single etch pit of the type described earlier and observed in the dissolution of AlGaAs heterostructures (Robertson and Fogler, 1996). The pit is the result of a layer-spanning defect with a radius of $2.1 \mu \mathrm{m}$ and a depth of $0.2 \mu \mathrm{m}$. The relevant parameters used in the simulations are summarized in Table 2 . The mass-removal rate is normalized by the nominal surface area, $L \times W$, to provide an average mass flux, $R$.

\section{Dissolution rate}

Figure 4A shows the comparison between discrete model and the analytical result for a single noninteracting etch pit (Robertson and Fogler, 1996). There are three transitions of interest marked on Figure 4: (1) the initial exposure of the substrate; (2) the beginning of pit interactions; and (3) the growth of the pit beyond the domain depth. Each of these transitions is depicted schematically in Figure 4B.

The first transition occurs when the epilayer is etched through, exposing the substrate. However, the model results show a delay between the initial exposure of the substrate and the corresponding rise in the average mass flux, $R$. This delay is due to the discrete nature of the domain. In a continuous domain, the pit begins to broaden immediately after exposure of the substrate. However, in a discrete domain

Table 1. Summary of the Effective Number of Exposed Faces

\begin{tabular}{cc}
\hline $\begin{array}{c}\text { Arrangement } \\
\text { Planar }\end{array}$ & $K$ \\
\hline 1 & 1 \\
$2{ }_{\perp}$ & $\sqrt{2}$ \\
$3_{\perp}$ & $\sqrt{3}$ \\
Kink & 2 \\
\hline
\end{tabular}

Table 2. Parameters Used in Simulations of Surface Evolution*

\begin{tabular}{llll}
\hline \multicolumn{1}{c}{ Parameter } & Value & Parameter & Value \\
\hline$s$ & $0.2 \mu \mathrm{m}$ & Substrate rate & $1.0^{-7}$ \\
Defect radius & $2.1 \mu \mathrm{m}$ & Epilayer rate & $1.0^{-11}$ \\
Defect depth & $0.2 \mu \mathrm{m}$ & Defect rate & $1.0^{-7}$ \\
Seconds per time step & $0.61 \mathrm{~s}$ & Reference rate & $1.2^{-6}$ \\
\hline
\end{tabular}

*All rates are reported in units of $\mathrm{mol} / \mathrm{cm}^{2} \cdot \mathrm{s}$.

broadening cannot begin until the exposed elements on the first level of the substrate are removed.

The second transition occurs when the pit in the discrete model begins to experience the effects of pit interactions. Even though there is only a single pit in the domain, pit interactions are possible due to the periodic boundary condi-

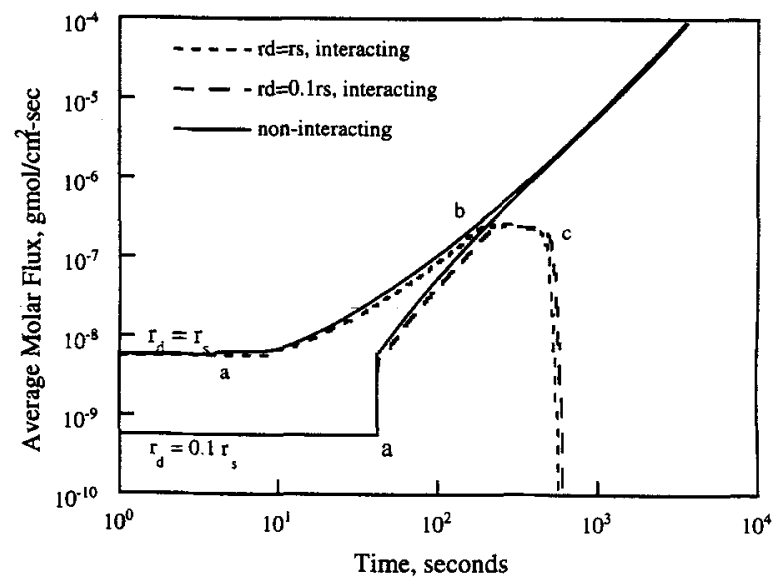

$\mathbf{A}$

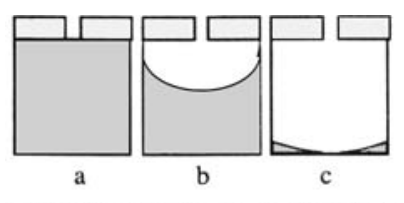

$\square$ SUBSTRATE $\square$ EPILAYER

B

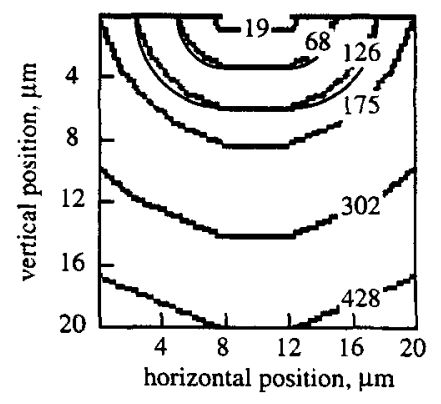

C

Figure 4. (A) Average mass fluxes predicted with discrete model vs. continuum model for noninteracting defects; (B) pit interactions resulting from periodic boundary conditions; (C) surface profiles along a cross section, including the center of the pit.

The labels are times in seconds. The solid lines at 68 and $126 \mathrm{~s}$ represent the profiles from the continuum model. 
tions imposed on the north-south and east-west faces. Thus this system represents a square array of defects.

The final transition occurs when the depth of the pit exceeds the depth of the domain. The pit can no longer grow in that direction and the exposed surface area decreases as the elements on the last level are removed. Consequently $R$ drops rapidly until the only elements remaining are those epilayer elements dissolving at the nondefect rate.

\section{Profiles of etched surfaces}

A profile of the etched surface is shown by Figure $4 \mathrm{C}$. The cross section used is in the $\langle 100\rangle$ direction and contains the center of the etch pit. The number of elements with exposed faces in a particular arrangement changes as the surface evolves over time. Figure 5 shows the number of elements with exposed faces in the $1,2_{\|}, 2_{\perp}, 3_{\perp}$ arrangements as a function of time. The number of elements 'with exposed faces in the remaining arrangements were insignificant compared to these four. The first vertical dotted line shows the time at which pit interaction begins; the second indicates the time at which the depth of the pit equals the depth of the domain.

\section{Simulated Dissolution of Systems with Multiple Defects}

The evolution of some surfaces with multiple defects can be simulated using a model system with a single defect and periodic boundary conditions. However, this approach is useful only when there is a very high degree of symmetry in the arrangement of the defects. For example, the situation discussed in the previous section used a square domain with a single pit located in the center of the domain. The pit grows to the edge of the domain where interactions begin simultaneously with the four first nearest neighbors. At no time did exactly two pits coalesce into a single pit and continue to grow as a unit. These results cannot be extrapolated to a more general situation, so systems with multiple defects must be studied explicitly.

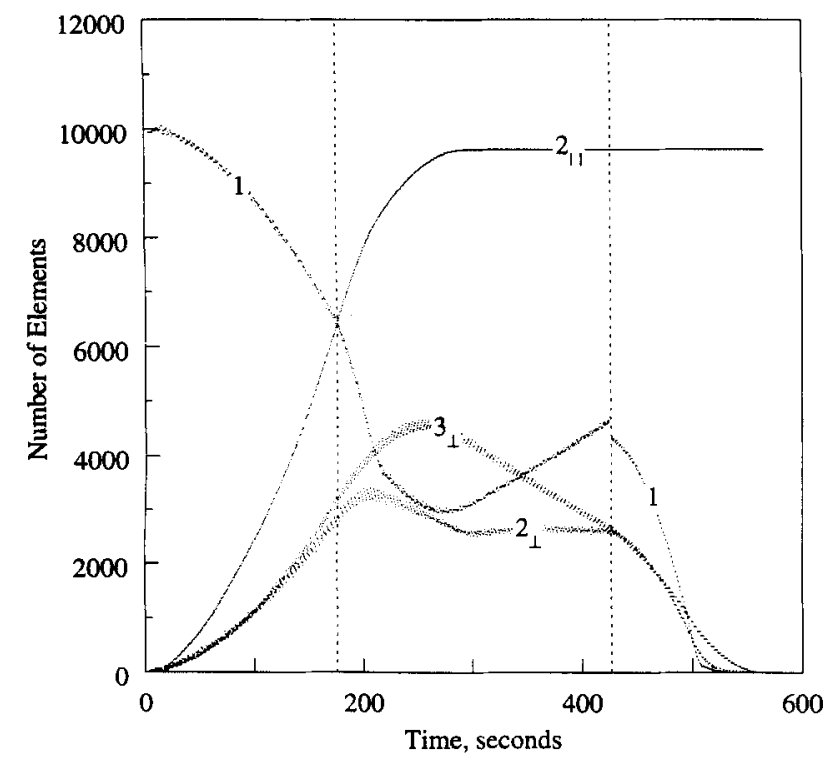

Figure 5. Number of elements with exposed faces in the $1,2_{\|}, 2_{\perp}$, and $3_{\perp}$ arrangements.
Systems of multiple defects are also relevant to a more interesting case: randomly arranged defects on a surface. An analytical analysis is limited to a system of noninteracting defects due to the intractability of accounting for areas and volumes held in common between multiple pits. This approach works well for a low density of pits during the initial stage of dissolution when pit interaction is minimal. However, simulations beyond that stage require a different approach.

\section{Two interacting defects}

There are several reasons to first consider the coalescence of two pits on a surface before attempting to describe the effects of higher densities of pits. First, the coalescence of two pits is the most likely multipit interaction at low etch-pit densities. Second, the trends observed for a two-pit interaction are instructive for interpreting the trends for higher etch-pit densities. The domain is 200 rows $\times 200$ columns $\times$ 100 levels with the first 10 levels composing the epilayer and the remaining 90 making up the substrate. The two defects are layer-spanning and have a diameter of 21 elements and a center-to-center distance of 50 elements. The edge length for each element is $0.2 \mu \mathrm{m}$.

The evolution of this surface undergoes several distinct stages: the dissolution of the defects, the growth of two individual pits, and the growth of the pits into each other. There are also two more stages that arise due to the finite domain depth and the periodic boundary conditions. The significance of these stages was discussed earlier, in the section describing the dissolution of individual isotropic etch pits. Figure 6 presents the simulation results for this system. Also included is the result of the corresponding noninteracting defects and the result of a single defect with the same initial surface area (i.e., $r^{\prime}=\sqrt{2} r$ ). The transition times indicated are (Figure 6A) the exposure of the substrate, (Figure 6B) the onset of pit coalescence, (Figure $6 \mathrm{C}$ ) the self-interaction due to the periodic boundary conditions, and (Figure 6D) the growth of the pit beyond the domain depth.

The most striking result from this simulation is that the pits coalesce into a structure that behaves in a fashion very similar to the noninteracting cases. The mass flux for two coalesced pits will eventually be almost indistinguishable from a single pit if they are allowed to grow unaffected by any additional interactions. This result suggests that clusters of pits will produce trends similar to those of individual pits if the clusters themselves are allowed to grow independently. The effect of higher defect densities on the surface evolution is treated in the following subsection.

\section{Systems of randomly placed defects}

Figure 7 shows the results for two simulations with randomly placed defects. The system parameters are identical to those of the previous case except the defects did not all span the epilayer, but rather the depths were randomly distributed according to a uniform distribution. Each defect covered approximately $3 \%$ of the domain surface. The defects were placed at random on the surface, and overlapping of the defects was allowed. Therefore, the actual surface coverage can be substantially less than the aggregate area of the individual defects. In fact, a fourfold increase in the number of pits led to only a $67 \%$ increase in the surface coverage, as seen from 


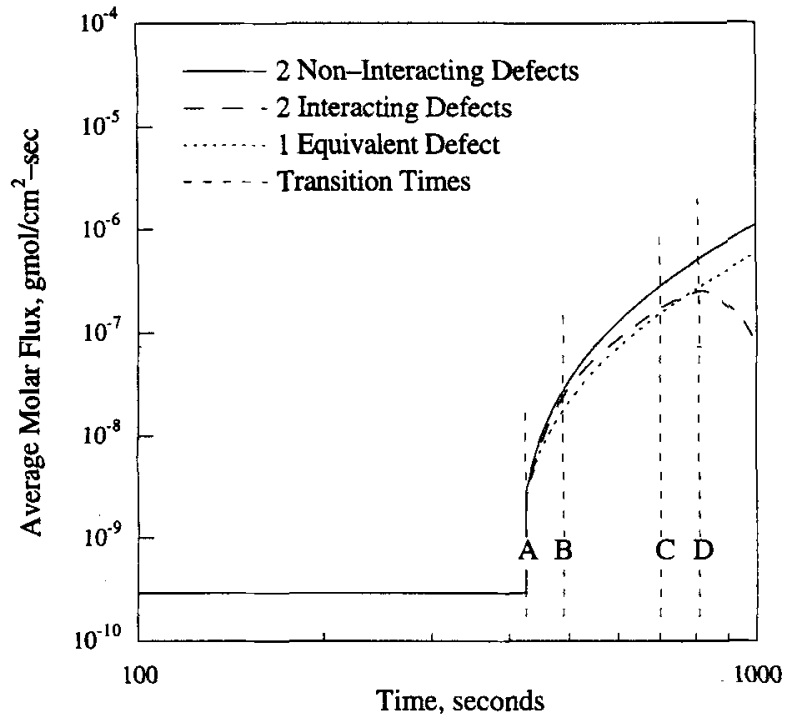

(a)

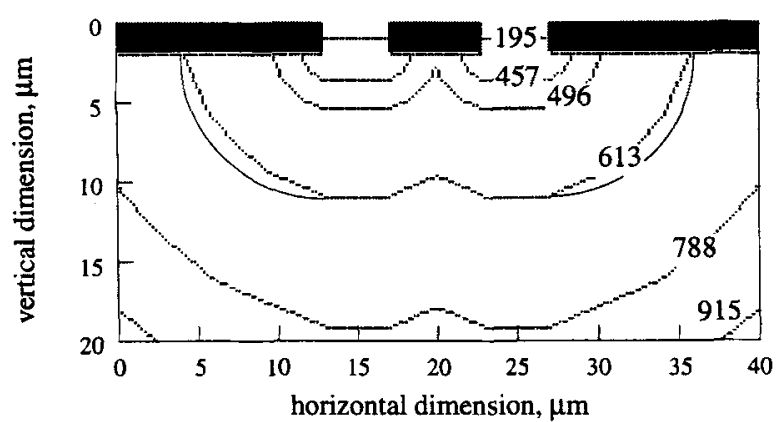

(b)

Figure 6. (A) Effects of interaction on the growth of two pits formed from two layer spanning defects with radius $2.1 \mu \mathrm{m}$ and a center-to-center distance of $10 \mu \mathrm{m}$; (B) profiles of the surface along a cross section $(i, 100, k)$, which includes the centers of both pits.

The corresponding time in seconds is indicated on the profile. The shaded regions denote the epilayer.

the change in the initial $R$ from Figure 7. Clusters of pits were apparent for the system with 25 defects. Periodic boundary conditions are enforced on the defects.

The dissolution behavior depicted in Figure 7 shows two primary stages of interest: before and after exposure of the substrate to the etchant at $426 \mathrm{~s}$. Before exposure, mass loss is due almost entirely to the dissolution of the defects. The nondefect rate is too low to make a significant contribution in either simulation. The variation in defect depths leads to a drop in $R$ before the substrate is exposed as the shallower defects are exhausted. The discrete jumps are due to the discretization of the epilayer into 10 levels.

After the exposure of the substrate to the etchant at $426 \mathrm{~s}$, $R$ jumps and then increases slightly as pits begin to form. The initial jump is, of course, due to the higher substrate rate; the continuing rise is due to the growth of the pit clusters under the epilayer. The amount of increase is limited by the time available for the growth of the cluster before interaction with the neighboring clusters.

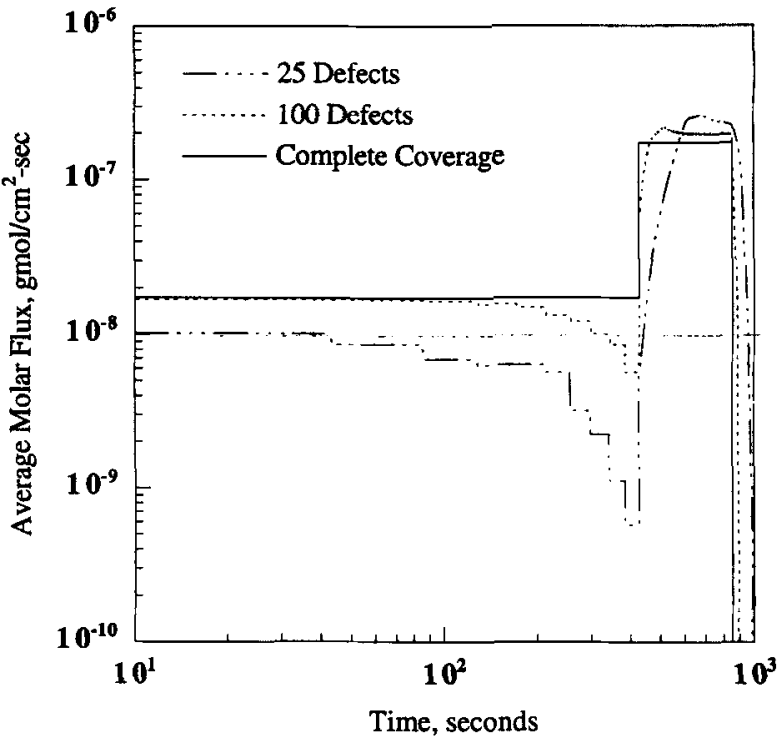

Figure 7. Simulation results for systems with 25 and 100 defects with a diameter of 21 elements placed at random on a 200 element by 200 element surface with uniformiy distributed depths.

The solid line is the limiting behavior expected for $100 \%$ coverage by layer-spanning defects.

\section{Conclusions}

This article presents a means of estimating the dissolution behavior of systems that are not easily rendered in a continuous model because of complicated geometries, etch rates that vary with position and/or orientation, or situations that couple the etch rate at the surface to properties of the bulk of the solid. The model can accommodate all of these circumstances by discretizing the surface into homogeneous, solid cubic volume elements and applying dissolution rules locally to each individual element. In addition, detailed knowledge of the orientation of the surface is not required by the dissolution rules.

The model was applied to systems where defects appeared in an otherwise uniform epilayer on a uniform substrate. The results agreed quantitatively with those of a case for which an analytical solution was available. The results also showed that clusters of interacting pits can produce trends in the average mass flux that are very similar to those observed for noninteracting pits. However, if the pits are arranged in a regular pattern, such as a square lattice, then there can be a significant change in the dissolution behavior of the surface. In fact, the overall etch rate will begin to decrease as the pits grow because of a loss of exposed surface area.

\section{Literature Cited}

Cale, T. S., and G. B. Raupp, "A Unified Line-of-Sight Model of Deposition in Rectangular Trenches," J. Vac. Sci. Technol. B, 8, 1242 (1990).

Cohen, N. S., "Review of Composite Propellant Burn Rate Modeling," ALAA J., 18, 277 (1980).

Cooke, M. J., and G. Harris, "Monte Carlo Simulation of Thin-Film Deposition in a Rectangular Groove,"J. Vac. Sci. Technol. A, 7, 3217 (1989). 
Dew, S. K., T. Smy, and M. J. Brett, "Simulation of the Microstructure of Chemical Vapor Deposited Refractory Thin Films," J. Vac. Sci. Technol. B, 10, 618 (1992).

Eden, M., Proc. Symp. on Information Theory in Biology, H. P. Yoekey, ed., Pergamon, New York, p. 339 (1958).

Hubbard, T. J., and E. K. Antonsson, "Emergent Faces in Crystal Etching," J. Microelectromech. Syst., 3, 19 (1994)

Ikegawa, M., and J. Kobayashi, "Deposition Profile Simulation using the Direct Simulation Monte Carlo Method,"J. Electrochem. Soc., 136, 2982 (1989)

Jaccodine, R. J., "Use of Modified Free Energy Theorems to Predict Equilibrium Growing and Etching Shapes,"J. Appl. Phys., 33, 2643 (1962).

Katardjiev, I. V., "A Kinematic Model of Surface Evolution During Growth and Erosion: Numerical Analysis," J. Vac. Sci. Technol. A, 7, 3222 (1989).

Meakin, P., "Fractal Scaling in Thin Solid Films," CRC Crit. Rev. Solid State Mat. Sci., 13, 143 (1987).

Meakin, P., T. Jфssang, and J. Feder, "A Two-Dimensional Reaction-Limited Selective Dissolution Model," Physica A, 196, 481 (1993).

Oian, S., R. C. Newman, R. A. Cottis, and K. Sieradzki, "Validation of a Percolation Model for Passivation of $\mathrm{Fe}-\mathrm{Cr}$ Alloys-Two Dimensional Computer Simulation," J. Electrochem. Soc., 137, 435 (1990).

Robertson, E. A., III, and H. S. Fogler, "The Reaction Kinetics of Photo-Active Defects in Semiconductor Dissolution," AIChE J., (1996).

Robertson, E. A., III, "The Dissolution of GaAs, AlGaAs, and Their Heterostructures," PhD thesis, Univ. of Michigan, Ann Arbor (1995).

Séquin, C. H., "Computer Simulation of Anisotropic Crystal Etching," Sens. Act. A, 34, 225 (1992).

Singh, V. K., and E. S. G. Shaqfeh, "Effect of Surface Re-Emission on the Surface Roughness of Film Growth in Low Pressure Chemical Vapor Deposition," J. Vac. Sci. Technol. A, 11, 557 (1993).

Thurgate, T., "Segment-Based Etch Algorithm and Modeling," IEEE Trans. Computer-Aided Des., CAD-9, 1101 (1991).

Watanabe, K., and H. Komiyama, "Micro/Macrocavity Method Applied to the Study of the Step Coverage Formation Mechanism of $\mathrm{SiO}_{2}$ Films by LPCVD,"J. Electrochem. Soc., 137, 1222 (1990).

Weeks, J. D., and G. H. Gilmer, "Dynamics of Crystal Growth," $A d$ vances in Chemical Physics, Vol. XL, I. Prigogine and Stuart A. Rice, eds., Wiley, New York (1978).

Wulu, H. C., K. C. Saraswat, and J. P. McVittie, "Simulation of Mass Transport for Deposition in Via Holes and Trenches," J. Electrochem. Soc., 138, 1831 (1991).

Yuuki, A., Y. Matsui, and K. Tachibana, "A Study on Radical Fluxes in Silane Plasma CVD from Trench Coverage Analysis," Japan J. Appl. Phys., 28, 212 (1989).

\section{Appendix}

The effective area, $K_{N, a}$, for elements associated with planes is computed based on the maximum projected area of the element. Planes that lie perpendicular to the principal vectors of the simple cubic geometry are exactly represented by elements with a single exposed face. Therefore, their projected area is $1 s^{2}$ per element so $K_{l}=1$.

Next consider resolving a section of a plane inclined at a $45^{\circ}$ angle using a single cubic element of the domain (refer to Figure 3). The actual area of the plane is $\sqrt{2} s^{2}$ per element. This is the area of the two exposed faces in the perpendicular arrangement projected along the normal vector of the plane, that is,

$$
2 s^{2} \frac{(1,1,0) \cdot(1,0,0)}{\sqrt{2}}=\sqrt{2} s^{2},
$$

Table A1. Average Number of Exposed Faces for All Arrangements (Kink and Planar)

\begin{tabular}{cccc}
\hline Arrangement & Area & $t^{\prime}$ & $K_{N, a}$ \\
\hline 1 & $s^{2}$ & $s / v$ & 1 \\
$2_{\|}$ & $2 s^{2}$ & $s / 2 v$ & 2 \\
$2_{\perp}$ & $2 s(s-v t)$ & $s / v$ & 1 \\
$3_{\|}$ & $2 s(s-v t)+s(s-2 v t)$ & $s / 2 v$ & 2 \\
$3_{\perp}$ & $3(s-v t)^{2}$ & $s / v$ & 1 \\
$4_{\|}$ & $4 s(s-2 v t)$ & $s / 2 v$ & 2 \\
$4_{\perp}$ & $2(s-v t)^{2}+2(s-v t)(s-2 v t)$ & $s / v$ & 2 \\
5 & $(s-2 v t)^{2}+4(s-v t)(s-2 v t)$ & $s / 2 v$ & 2 \\
6 & $6(s-2 v t)^{2}$ & $s / 2 v$ & 2 \\
\hline
\end{tabular}

which implies $K_{2, \perp}=\sqrt{2}$. A similar argument made for the $3_{\perp}$ arrangement produces

$$
3 s^{2} \frac{(1,1,1) \cdot(1,0,0)}{\sqrt{3}}=\sqrt{3} s^{2}
$$

and subsequently $K_{3, \perp}=\sqrt{3}$.

For elements associated with kinks, the effective area is based on the time-averaged area of the element. The average can be calculated assuming exposed surfaces etch uniformly in the direction opposite the surface normal, receding with a velocity, $v$, given by $M w r / \rho$ (unexposed faces remain stationary).

The element represents a volume in space bounded by six planes that correspond to the faces of the element. The element, which was initially a cube, is effectively being reduced in size. The geometry remains a rectangular prism, though not necessarily a cube. The surface area changes with time in a definite fashion, thus providing a means to calculate the average area, which is taken to be the effective area used in Eq. 2:

$$
K_{N, a} \equiv \frac{1}{s^{2} t^{\prime}} \int_{0}^{t^{\prime}} A(t) d t
$$

where $t^{\prime}$ is the time required to completely dissolve the element, $A(t)$ is the surface area of the prism, and $s$ is the initial edge length of the cubic element.

An instructive example is the case where all six faces of an element are exposed to the etchant. As each face is dissolved, it moves toward the geometric center of the element with a velocity $v t$. The edge length varies linearly with time according to $(s-2 v t)$; the total exposed surface area, $A(t)$, is $6(s-$ $2 v t)^{2}$; and the time required to dissolve the element, $t^{\prime}$, is $s / 2 v$. Thus,

$$
K_{6}=\frac{1}{s^{2} t^{\prime}} \int_{0}^{t^{\prime}} 6(s-2 v t)^{2} d t=2 .
$$

The same procedure is applied to the remaining elements. Table A1 lists $A(t), t^{\prime}$, and $K_{N, a}$ for each arrangement. The analysis was also applied to the planar elements for the purposes of comparison.

Manuscript received Nov. 3, 1995, and revision received Mar. 27, 1996. 